\title{
Lupus Membranous Nephropathy
}

\author{
Claudio Ponticelli ${ }^{\mathrm{a}}$ Gabriella Moroni ${ }^{\mathrm{b}}$ Alessia Fornoni ${ }^{\mathrm{c}}$

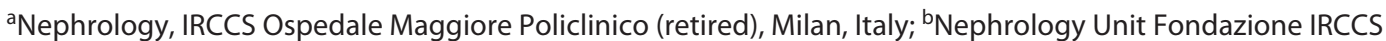 \\ Ca' Granda Ospedale Maggiore Milano, Milan, Italy; 'Katz Family Division of Nephrology and Hypertension and \\ Peggy and Harold Katz Family Drug Discovery Center, University of Miami, Miami, FL, USA
}

\section{Keywords}

Glomerular diseases · Membranous nephropathy · Lupus nephritis · Nephrotic syndrome · Chronic kidney disease

\section{Abstract}

Background: Lupus membranous nephropathy (LMN) is a rare disease, usually associated with nephrotic syndrome. Methods: We reviewed the literature by searching for the following terms on Pubmed.gov: lupus nephritis, membranous nephropathy (MN), lupus membranous nephropathy, nephrotic syndrome, and Class V lupus nephritis. Results: The histology of LMN at light microscopy is similar to that of primary MN. Cases of MN associated with focal or diffuse proliferation are not considered LMN by the International Society of Nephrology/Renal Pathology Society classification. Immunofluorescence study of LMN shows deposits of all immunoglobulins and complement. Tubulo-reticular structures, extraglomerular deposits, subepithelial, and scanty subendothelial deposits can be seen on electron microscopy. Phospholipase A2 receptor deposits are usually but not necessarily absent in LMN. The pathogenesis is still not completely understood. The inflammatory milieu of lupus may favor the development of autoantigens and intraglomerular assembly of immune complexes. These are more often associated with mesangial or endocapillary hypercellular lesions. Alternatively, autoantibodies may bind autoantigens in the glomerular subepithelium, triggering a signaling cascade leading to LMN. A central role in the development of podocyte injury and proteinuria is played by the components of complement C5b-C9. CKD progression in LMN is slow but may be accelerated by the frequency of renal flares. Persistent nephrotic syndrome and/or the frequent use of corticosteroids may lead to a series of life-threatening complications. Discussion: Treatment of arterial hypertension, dyslipidemia, and diabetes are of paramount importance. Besides specific therapies of these complications, hydroxychloroquine and vitamin D supplementation are recommended. Immunosuppression should be limited to patients with nephrotic proteinuria. The most frequently used drugs are corticosteroids, calcineurin inhibitors, cyclophosphamide, mycophenolate, and rituximab, alone or combined. Early detection and treatment of renal flares is of paramount importance to prevent CKD progression.

(C) 2021 The Author(s)

Published by S. Karger AG, Basel

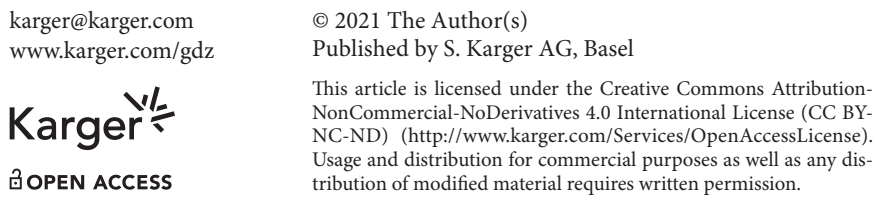

karger@karger.com www.karger.com/gdz

Karger $\stackrel{\text { ' }}{=}$

BOPEN ACCESS

(C) 2021 The Author(s)

Published by S. Karger AG, Basel

This article is licensed under the Creative Commons AttributionNonCommercial-NoDerivatives 4.0 International License (CC BYNC-ND) (http://www.karger.com/Services/OpenAccessLicense) Usage and distribution for commercial purposes as well as any distribution of modified material requires written permission.

Claudio Ponticelli 


\section{Introduction}

Renal disease is a frequent and severe cause of morbidity in systemic lupus erythematosus (SLE). Lupus membranous nephropathy (LMN), which is an uncommon subtype of lupus nephritis, is usually associated with nephrotic syndrome and can have a variable course; some patients maintain stable kidney function or show a slow progression, while others can have renal flares and transformation to more severe proliferative forms eventually leading to ESRD or to life-threatening extrarenal complications, including thrombotic events, cardiovascular disease (CVD), or infections. In many cases, a combination of membranous and proliferative lesions may be seen in renal biopsies. In this review, cases of mixed membranous and proliferative lupus nephritis will not be considered. Only LMN, in line with the current classification, will be discussed.

\section{Pathology}

The old World Health Organization (WHO) histological classification of lupus nephritis identified 6 subclasses of nephritis: I normal glomeruli, II pure mesangial alterations, III focal segmental glomerulonephritis, IV diffuse glomerulonephritis, $\mathrm{V}$ diffuse membranous glomerulonephritis, VI advanced sclerosing glomerulonephritis [1]. Class V was subdivided into: pure LMN (Va), LMN associated with mesangiopathy ( $\mathrm{Vb}), \mathrm{LMN}$ with signs of focal segmental glomerulonephritis (Vc), and LMN associated with diffuse glomerulonephritis (Vd). The currently used classification of the International Society of Nephrology and the Renal Pathology Society also divides lupus nephritis into 6 classes but provides an improved description of the various lesions and classes, allowing for a better standardization. In particular, class $\mathrm{V}$ is defined as membranous lupus nephritis with global or segmental continuous granular subepithelial immune deposits, often with concurrent mesangial immune deposits. Any degree of mesangial hypercellularity may occur in class $\mathrm{V}$ [2]. If present under light microscopy, subendothelial deposits warrant a combined diagnosis of lupus nephritis class III and V, or class IV and V, depending on their distribution.

LMN presents with features similar to the primary form when investigated by light microscopy (Fig. 1). In many cases, no definite etiology may be recognized on the basis of histological findings even though mesangial proliferation may be seen in LMN (Fig. 2). However, a com-

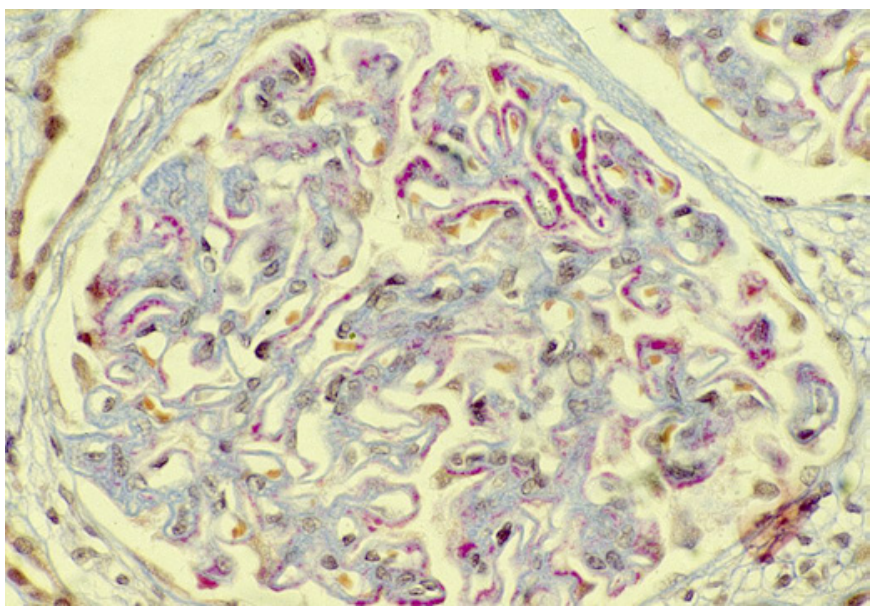

Fig. 1. Renal biopsy in a patient with LMN. AFOG stain of one glomerulus with diffuse thickening of glomerular basement membrane associated with the presence of many granular immune deposits in subepithelial position in a case of class V lupus nephritis. LMN, lupus membranous nephropathy.

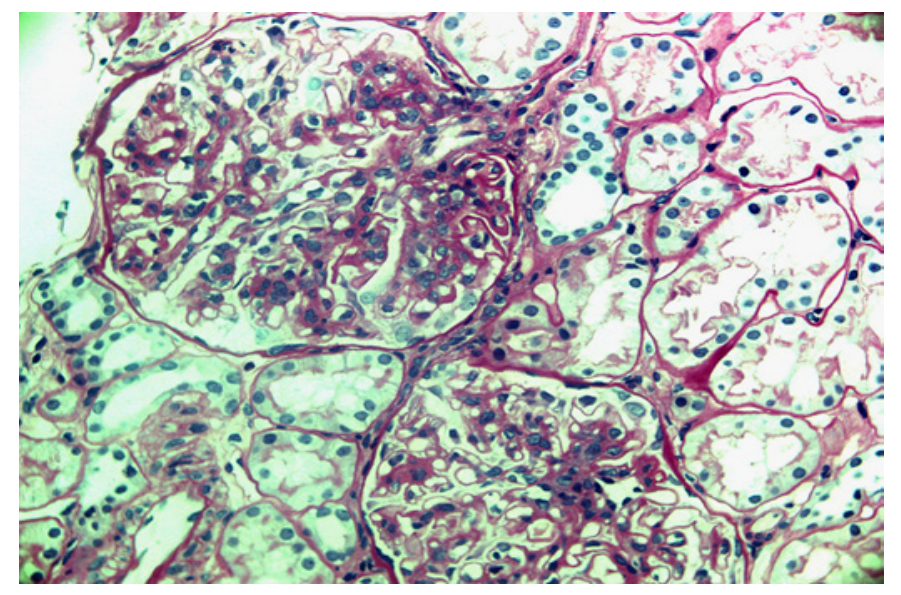

Fig. 2. Light microscopy. Periodic acid-Schiff stain of 2 glomeruli with thickened of the capillary walls associated with areas of mesangial proliferation in a patient with lupus nephritis.

bination of immunofluorescence staining and structural changes by electron microscopy can distinguish LMN from primary $\mathrm{MN}$ with high specificity and varying sensitivity. By immunofluorescence, the most important features of LMN are "full-house" glomerular deposits of IgG, $\operatorname{IgM}$, IgA, and intense $\mathrm{C} 1 \mathrm{q}, \mathrm{C} 3$, and $\mathrm{C} 4$ staining in the subepithelial space (Fig. 3) and occasionally in the mesangium. The presence of prominent deposits of IgG 1-3 may also distinguish LMN from primary $\mathrm{MN}$, in which deposits of IgG4 are predominant [3]. Complement and IgG deposits on tubular basement membranes and on 
vessel walls can also be seen in LMN. Of importance, using direct immunofluorescence microscopy of frozen tissue or paraffin-immunofluorescence of pronase-digested specimens, epitopes of the phospholipase A2 receptor (PLA2R) protein are found to be overexpressed in glomeruli in about $70 \%$ of patients with primary MN [4]. Such deposits have a pattern similar to the IgG deposits and may persist for long periods (weeks and months) after the immune activity of the disease has waned [5]. In some cases of secondary MN (most notably sarcoidosis, cancer, and hepatitis B viral infection) including LMN, hyperexpression of PLA2R antigen, or circulating anti-PLA2R antibodies can be seen [6]. On the contrary, while in up to $5 \%$ of patients with primary $\mathrm{MN}$ deposits of thrombospondin type-1 domain-containing 7A (THSD7A) can be detected [7], none have been described in LMN. This is also the case for 2 newly recognized autoantigens such as exostosin and neural epidermal growth factor-like 1 protein.

Electron microscopy confirms the presence of subepithelial deposits (Fig. 4). Scattered subendothelial immune deposits may be also identified in electron microscopy. Tubulo-reticular structures ("interferon-fingerprints") in endothelial cells, extraglomerular deposits, combined subendothelial, and subepithelial deposits have also been reported.

A modified NIH activity index has been proposed to monitor the severity of lupus nephritis [8]. However, important components of this index are not observed in LMN, including endocapillary proliferation, fibrinoid necrosis, and crescents. Clinical features, such as degree of proteinuria, RBC, and white blood cell count have been proposed to measure disease activity [9]. On the contrary, chronic lesions associated with LMN such as global sclerosis, tubular atrophy, and interstitial fibrosis can be easily captured by the chronicity index. Interestingly, tubulointerstitial inflammation and tubulointerstitial fibrosis are associated with increased risk of renal flares in patients with LMN, thus stressing the role of innate immunity in the pathogenesis of LMN [10].

\section{Pathogenesis}

Few data exist about the pathogenesis of LMN and how it differs from other forms of lupus nephritis and from primary MN. The subepithelial distribution of immune complex deposits suggests that similar mechanisms are involved in $\mathrm{LMN}$ when compared to primary $\mathrm{MN}$ [11], although important differences remain. In primary

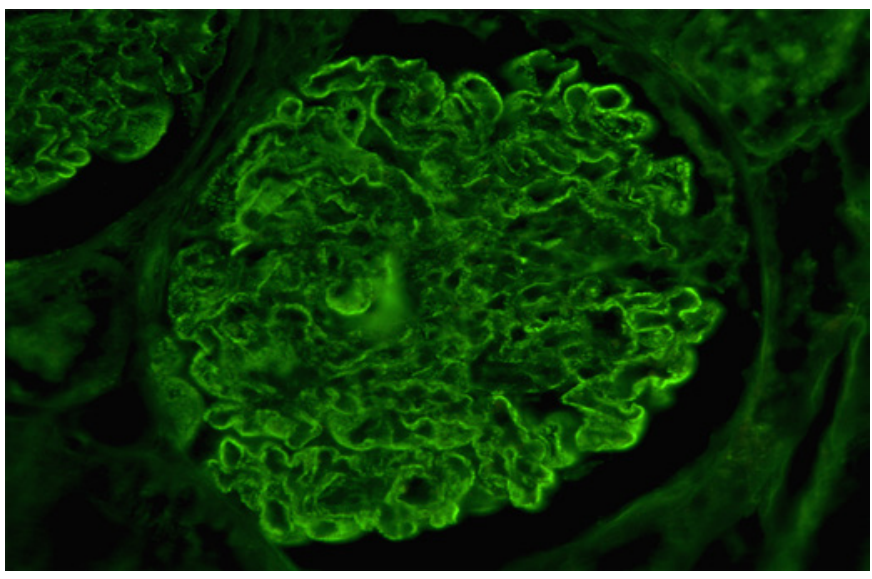

Fig. 3. Immunofluorescence: strong and diffuse granular IgG deposits along the glomerular basement membrane in a case of class V lupus nephritis.

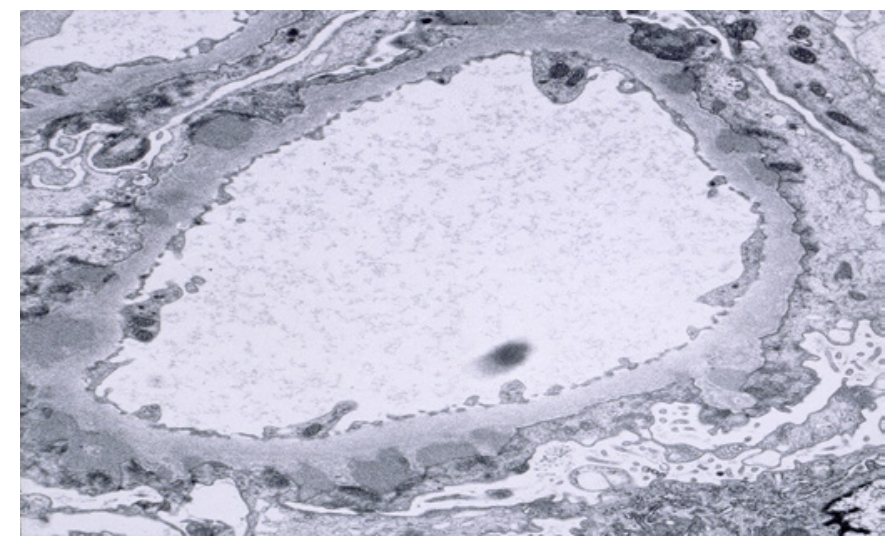

Fig. 4. Electron microscopy of glomerular capillary reveals variably sized subepithelial electron-dense deposits.

$\mathrm{MN}$, endogenous or external (virus?) stimuli may increase the expression of PLA2R1 epitopes or shed some "cryptic" epitopes of PLA2R [12], which are located in the subepithelial space of glomerular basement membrane (GBM). In lupus nephritis, autoantigens are produced by inefficient apoptosis and neutrophil extracellular traps (NETs). Defective apoptotic clearing can allow nucleosomes normally contained within glomerular cells to become renal autoantigens and can cause antigen modifications, such as nucleic acid oxidation, which increases the inflammatory properties of self-antigens [13]. NETosis is a specialized form of cell death. Proteins derived from NET may also serve as self-antigens, which accumulate and may contribute to the development of renal lesions and to disease severity in SLE [14]. As circulating free
12

Glomerular Dis 2021;1:10-20

DOI: $10.1159 / 000512278$
Ponticelli/Moroni/Fornoni 


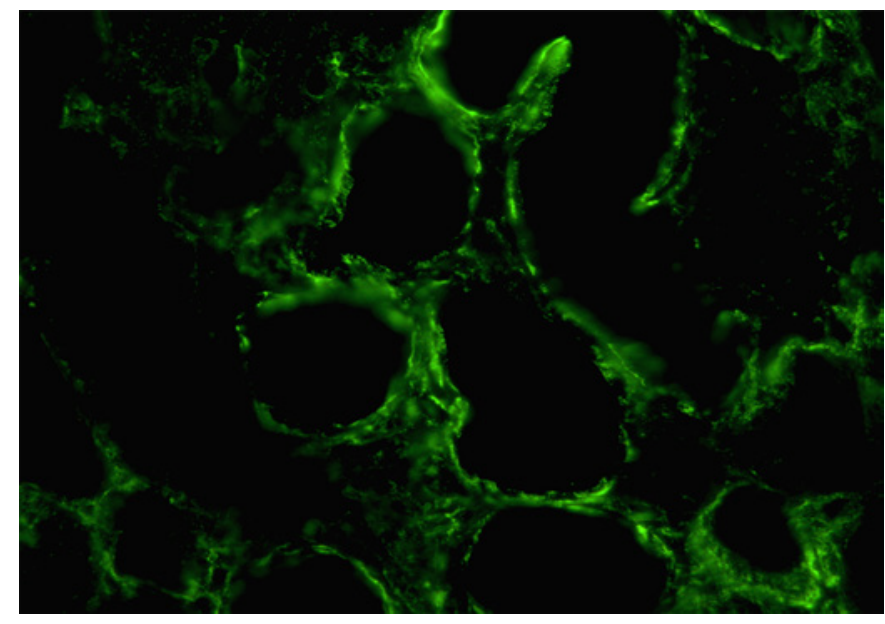

Fig. 5. Granular deposits of IgG along the tubular basement membranes.

DNA has been reported in patients with lupus nephritis and recognized as the triggering event for the generation of anti-dsDNA antibodies [15], and dsDNA can activate cGAS/STING signaling pathways [16], the possibility of a ds-DNA/STING signaling activation as a contributor to the development of LMN deserves further investigation. In primary $\mathrm{MN}$, autoantibodies to PLA2R, usually of IgG4 subclass, bind to conformational epitopes on specific domains of PLA2R expressed on the podocyte surface, form local immune complexes in situ and activate complement by the mannan-binding of complement cascade [17]. Different districts of the glomerulus may be affected by the immunological response in LMN, including podocytes [18], tubular basement membranes (Fig. 5), and tubulointerstitium [19]. There are no doubts that nucleosomes are central targets for nephritogenic antibodies in lupus nephritis [20] but how subepithelial immune deposits may form in LMN is still uncertain. Ma et al. [21] suggested 3 possible mechanisms: (1) subepithelial deposition of preformed circulating immune complexes. Lowaffinity antigen-antibody interactions may allow immune complexes initially trapped in a subendothelial position to dissociate and reform in the subepithelial space of GBM. (2) Intrinsic glomerular antigens that accumulate in the subepithelial space and serve as target antigens for circulating antibodies. (3) Histone-rich nucleosomes can traverse the GBM due to electrostatic interactions and remain planted in the subepithelial space [22]. Whatever the mechanism, autoantigens located in the subepithelial space of the GBM are targeted by autoantibodies, which are mainly IgG1, 2, or 3 at difference with primary MN.
In addition, the dominant pathway of complement activation in lupus nephritis is the classical one, even though activation of the alternative pathway may also play a role [23]. The local formation of immune complexes can cause podocyte injury and trigger a sequence of events similar to that observed in primary $\mathrm{MN}$, as postulated by Austin and Illei [11]. The membrane attack complex (MAC) is the main cause of proteinuria in primary MN. MAC is the acronym to indicate the assembly of the late components of complement, namely, the complex C5b-9. In sublytic quantities, C5b-9 can stimulate podocytes to produce a number of inflammatory mediators - such as prostanoids, proteases, cytokines, extracellular matrix components, and reactive oxygen species - that may impair the filtration barrier by peroxidation of membrane proteins and collagen. C5b-9 can also alter the actin cytoskeleton. In response to a C5b-9 attack, the glomerular epithelial cells activate specific signaling pathways that may cause partial dissolution of the actin cytoskeleton, reduce nephrin expression, reduce F-actin-bound nephrin, and loss of slitdiaphragm integrity. On the other hand, signals such as endoplasmic reticulum stress may limit complement-induced injury or promote recovery [24]. The extent of complement activation and glomerular injury is dependent, in part, on complement-regulatory proteins, which act at early or late steps within the complement cascade. Complement components in proteinuric urine also induce tubular epithelial cell injury and mediate progressive interstitial disease with consequent abnormal distribution of slit-diaphragm protein and detachment of podocytes that are shed into Bowman's space, where they produce cell activation, by converting normal cells into resident inflammatory effector cells that cause injury [25]. Experimental studies supporting a role of MAC in LMN are lacking, but it is reasonable to speculate that in the inflammatory environment of lupus nephritis both classical and alternative pathways of complement are activated, leading to activation of C5 convertase and MAC. The consequent alterations of the GBM and the protein filtration barrier of podocytes eventually result in proteinuria (Fig. 6). However, these studies have been mainly conducted in animals that developed proliferative lupus nephritis. Only one study in MRL/lpr mice showed that Th1 responses depend on the function of the WSX-1 gene, which encodes a subunit of the IL-27R with homology to IL-12R. Mice deficient for the WSX-1 gene developed disease resembling human membranous glomerulonephritis with a predominance of IgG1 in glomerular deposits, accompanied by increased IgG1 and IgE in the sera [26]. 


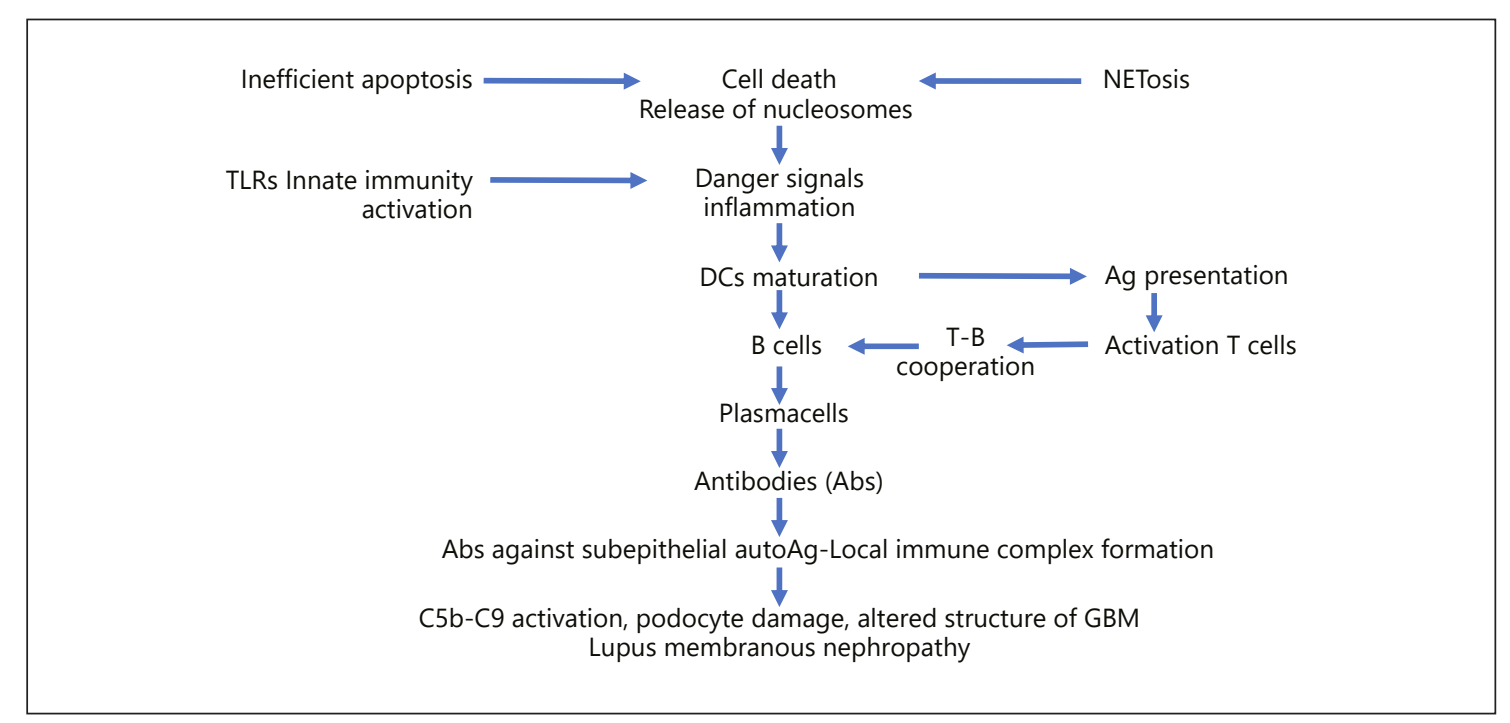

Fig. 6. A schematic view of possible pathogenesis of LMN. Defective apoptosis and NETosis can release nucleosomes from dead cells, which are considered as self-antigens. In LMN, it is possible that nucleosomes directly accumulate in the subepithelial space or derive from circulating immune complexes that dissociate on the subluminal side of GBM or cross the GBM and plant in the subepithelial space (see text). The location of these new antigens elicits the production of autoantibodies, mainly IgG 1-3 with local formation of immune complexes and activation of complement,

\section{Clinical Presentation}

Pure LMN accounts for approximately $15-20 \%$ of lupus nephritis cases [27]. Like most forms of SLE, LMN also mainly affects females. The mean age at presentation ranges around 35 years. Children may also be affected by pure lupus $\mathrm{MN}$.

The clinical presentation is variable both in children and adults. Many patients do not have extrarenal signs or symptoms of SLE and present proteinuria and abnormal urinary sediment as the sole disease manifestation, antedating other clinical features and even immunological markers of the disease by years. Proteinuria is the hallmark of the disease. Many patients have a full blown nephrotic syndrome with proteinuria exceeding $3.5 \mathrm{~g}$ per day, hypoalbuminemia, dyslipidemia, and variable degrees of edema. Hematuria and hypertension are common. However, other patients may be asymptomatic and are discovered to be affected by the disease only at routine check-up visits or by incidental urinalyses. Renal function is usually normal or subnormal. In a few patients with severe nephrotic syndrome LMN may be detected for the first time because of thrombotic events. by the classical and alternative pathways, leading to the production of $\mathrm{C} 5$ convertase that cleaves $\mathrm{C} 5$ into $\mathrm{C} 5 \mathrm{a}$ and $\mathrm{C} 5 \mathrm{~b}-\mathrm{C} 9$. The anaphylatoxin $\mathrm{C} 5 \mathrm{a}$ can also recruit neutrophils and macrophages, while the MAC C5b-C9 results in podocyte damage and excessive matrix material that alters the GBM, leading to proteinuria and features of membranous nephropathy. Abs, antibodies; LMN, lupus membranous nephropathy; NET, neutrophil extracellular trap; GBM, glomerular basement membrane; MAC, membrane attack complex.

\section{Outcome and Prognosis}

The outcome of LMN may be diverse. Both spontaneous remission of proteinuria and transformation from LMN to proliferative lupus nephritis class III or IV are well documented [28, 29]. Only few studies report longterm follow-up. An American study showed that most of 36 patients with LMN WHO class $\mathrm{Va}$ or $\mathrm{Vb}$ maintained proteinuria over time but about $28 \%$ developed ESRD within 10 years from diagnosis. Initial serum $\mathrm{Cr}$ was the only independent risk factor that predicted ESRD [30]. In a Chinese trial, 38 patients with WHO class Va (45\%) or $\mathrm{Vb}(55 \%)$ treated with prednisone and azathioprine were followed for a mean of 90 months. The cumulative risk of renal relapse was $12 \%$ at 36 months and $16 \%$ at 60 months. During the follow-up 13\% of patients had a decline of $\mathrm{Cr}$ clearance by $20 \%$, but none had doubling of serum Cr. Renal outcome was not significantly worse in patients presenting with nephrotic syndrome [31]. A multicenter French study reported the outcome of 66 participants with a mean age of 31 years, followed for a mean period of $6.9 \pm 0.2$ years. At 10 years a transition from LMN to proliferative nephritis was seen in 14 patients $(21 \%), 4$ of 
them progressed to ESRD. Four other patients entered ESRD, 2 because of biopsy proven progressive fibrosis. Renal survival at 10 years was $88 \%$, but it fell to $50 \%$ in the few patients followed for 20 years. Nephrotic syndrome increased the risk of ESRD, but at multivariate analysis it did not represent an independent risk factor for kidney function deterioration. Thirty-four patients (51\%) experienced sustained renal remissions. Thrombosis occurred in 15 patients $(23 \%)$ and was more frequent in those with nephrotic syndrome [32]. An Italian multicenter study reported the outcome of 67 patients with pure LMN. After a mean follow-up of $13 \pm 8.6$ years, $86 \%$ of patients who received different types of treatment were in complete or partial remission. The probability of being alive with kidneys functioning at 10 years was also $86 \%$. Five patients with pure LMN died after a follow-up ranging from 130 to 298 months. Causes of death were sepsis in one case, cardiac arrest in another patient and unknown causes in 3 patients. At multivariate analysis, initial serum $\mathrm{Cr}$, chronicity index, nephritic flares, and failure to respond to treatment were independent predictors of renal failure [33].

It appears from these studies that the progression of the disease is usually slow but can be accelerated by transformation to a more severe class or by renal flares. Membranous transformation to proliferative glomerulonephritis is common and can lead to significant changes in the outcome and treatment of lupus nephritis. Renal flares may be subdivided into proteinuric flares, characterized by increase in proteinuria with stable kidney function, and nephritic flares, characterized by a substantial increase in serum Cr [34]. Nephritic flares are difficult to manage and may lead to irreversible lesions, while proteinuric flares usually respond to treatment, but the duration of flare is an independent predictor of CKD [35]. In addition, flares in patients with lupus $\mathrm{MN}$ are frequently associated with conversion to proliferative glomerulonephritis, as shown by renal biopsy [36]. Thus, prompt diagnosis and treatment of renal flares are critical to prevent irreversible lesions.

\section{Several Other Risk Factors May Concur in Causing Complications in Patients with LMN}

In spite of the young age at diagnosis, patients with LMN are prone to CVD and thrombotic complications, which are often triggered by hypoalbuminemia and/or corticosteroids. Arterial hypertension is frequent in patients with SLE and can contribute significantly not only to accelerated atherosclerosis, and CVD but also to CKD. Dyslipidemia, diabetes, and hypercoagulability are other risk factors for CVD and thrombosis. High serum levels of total cholesterol and triglycerides are commonly found to accompany glomerular disease, especially in those situations associated with heavy proteinuria and hypoalbuminemia or in patients who received high doses or prolonged use of corticosteroids. The importance of high LDL-cholesterol as a risk factor for CVD is well established in the general population; the combined measure of LDL- and VLDL-cholesterol, that is, cholesterol carried by apoB particles, seems to be an even better marker for atherosclerosis. Corticosteroids may also induce insulin resistance and favor the development of type 2 diabetes. Patients with nephrotic syndrome are at increased risk for venous and or arterial thrombotic or embolic events, triggered by a combination of increased plasma levels of fibrinogen, low albumin levels, urinary loss of protein $\mathrm{C}$, protein $\mathrm{S}$, antithrombin III, and hyperviscosity favored by hypercholesterolemia [37]. The presence of antiphospholipid antibodies may also predispose to arterial and venous thrombosis.

\section{Symptomatic Therapy}

Prevention or early treatment of the complications reported above is critical to improve the life-expectancy and the quality of life of lupus patients with MN. There is a general agreement that renin-angiotensin system (RAS) inhibitors should be the preferred drugs to manage arterial hypertension. These agents can reduce blood pressure and can also possess antiproteinuric effects. Calcium channel blockers with or without diuretics are the second option for treating hypertension in patients who do not respond to RAS inhibitors. Thiazides are also used to better control blood pressure and to prevent edema. However, in case of severe edema, loop diuretics are needed. Oral or intravenous furosemide is the loop diuretic more frequently used in spite of its variable bioavailability. If high-dose furosemide fails to reduce edema, combination with a thiazide diuretic is usually attempted. In few nephrotic patients with furosemide-resistant edema, tolvaptan or amiloride can be used with success.

Statins are the drugs of choice to treat hypercholesterolemia. These lipid-lowering agents are usually well tolerated but at high doses they can cause myopathy and even rhabdomyolysis. Caution should be used in prescribing statins to patients taking cyclosporine. This drug can increase the risk of statin side effects by inhibiting the activ- 
ity of cytochrome P 3A4, a regulator of the cell influx and efflux of statins. Ezetimibe, alone or on top of statins, is suggested for severe hypercholesterolemia, although the evidence base for this recommendation is weak in LMN. New lipid-lowering drugs are emerging but their efficacy and safety in patients with glomerular diseases still need to be assessed.

Avoiding smoke, soft beverages and alcohol, reducing carbohydrates in the diet, and regular physical activity may help reduce the risk of developing type 2 diabetes. Probably the most important measure is a consistent reduction of the body weight. Sodium-glucose cotransporter 2 inhibitors are good candidates for preventing diabetes complications. They may prevent major adverse cardiovascular events and progression of renal disease [38]. Vitamin D deficiency is frequent both in nephrotic patients and in those with SLE. Such a condition may be involved in the development of rickets and osteomalacia, in the progression of CVD and in an increased susceptibility to infections. A significantly inverse relationship between 25-hydroxyvitamin D D levels and lupus activity has also been reported [39]. Thus, in nephrotic patients, serum levels of dihydroxyvitamin D are worth to be measured. Supplementation should be prescribed in case of low levels. A total vitamin D supply of $100 \mu \mathrm{g}(4,000 \mathrm{IU}) /$ day is required to reach serum dihydroxyvitamin $\mathrm{D}$ concentrations $>100 \mathrm{nmol} / \mathrm{L}$. There is no evidence of adverse effects with serum concentrations $<140 \mathrm{nmol} / \mathrm{L}$. Published cases of vitamin D toxicity with hypercalcemia, for which the dihydroxyvitamin $\mathrm{D}$ concentration and vitamin $\mathrm{D}$ dose were known, all involved intakes of $\geq 1,000 \mu \mathrm{g}$ [40].

The use of anticoagulation to prevent thrombotic events in patients with full blown nephrotic syndrome is still discussed. Years ago, a decision analysis in patients with primary $\mathrm{MN}$ concluded that warfarin can reduce the risk of thromboembolic more than it increases the risk of hemorrhagic complications [41]. Direct oral anticoagulants (DOACs) have been largely used in kidney diseases, but there is little information about their efficacy in patients with nephrotic syndrome. Most direct oral anticoagulants are cleared by the kidney and circulate bound to proteins; thus, the pharmacokinetics of these drugs can be influenced by reduced renal function and proteinuria [42]. The KDIGO 2012 guidelines is to consider full-dose anticoagulation if serum albumin drops below $2.0-2.5 \mathrm{~g} /$ dL [43]. Low-dose aspirin has also been proposed for nephrotic patients with good levels of albuminemia [44]. At present, prophylactic anticoagulation may be suggested for patients with a previous history of venous thrombo- embolic events, and for those with additional risk factors (hereditary thrombophilic predisposition, immobility, obesity, and anti-phospholipid antibodies positivity).

Hydroxychloroquine (HCQ) is an inexpensive drug, although the price is variable depending on country. HCQ may permit reduced dosing for corticosteroids and immunosuppressive drugs in lupus nephritis. HCQ may also prevent the lupus flares and the thrombotic complications in patients with anti-phospholipid syndrome. Further benefits may include protection against osteoporosis and atherosclerosis. HCQ is recommended in lupus patients by the recent guidelines of the Joint European League Against Rheumatism and European Renal Association-European Dialysis and Transplant Association [45]. With proper dosing and monitoring, HCQ is generally a safe drug. HCQ dosing is weight based (usually $5 \mathrm{mg} / \mathrm{kg} /$ day) and may need to be adjusted in advanced CKD. However, like any other medication, HCQ can be responsible for a number of adverse events. HCQ side effects are usually dose-dependent and many adverse events reflect intentional or unintentional over-dosage. Children are particularly vulnerable to side effects of chloroquine. Given its similarity to chloroquine, also HCQ should be considered potentially toxic in the pediatric population. The most severe complication is retinopathy. High-dose and long-use duration are the most significant risks of developing retinopathy. The risk dramatically increases with cumulative doses higher than 1,000 g of HCQ. A yearly annual ophthalmological examination is recommended. Cardiotoxicity is a rare but serious complication of HCQ. It commonly manifests as a restrictive or dilated cardiomyopathy or with conduction system abnormalities including atrioventricular block and bundle branch block. Risk factors for the development of HCQinduced cardiotoxicity include older age, female gender, longer duration of therapy ( $>10$ years), elevated per-kilogram daily dose, preexisting cardiac disease, and renal insufficiency [46].

\section{Immunosuppressive Treatment}

There is agreement that patients with persistent nephrotic syndrome in spite of the use of RAS inhibitors should receive immunosuppressive therapy, while there is controversy about the use of immunosuppression in patients with subnephrotic proteinuria. The Joint European League Against Rheumatism and European Renal Association-European Dialysis and Transplant Association (EULAR/ERA-EDTA) recommended the use of cor-
16

Glomerular Dis 2021;1:10-20 DOI: $10.1159 / 000512278$
Ponticelli/Moroni/Fornoni 
ticosteroids and immunosuppressive drugs in pure class $\mathrm{V}$ nephritis, when the ratio urine protein/Cr exceeds $1,000 \mathrm{mg} / \mathrm{g}$ despite the optimal use of renin-angiotensinaldosterone system blockers [45].

Several immunosuppressive agents have been used in LMN, including corticosteroids, purine synthesis inhibitors, alkylating agents, or calcineurin inhibitors (CNIs) either alone or associated with corticosteroids and/or purine synthesis inhibitors (multi-target therapy). Retrospective studies in patients with LMN and nephrotic syndrome reported the efficacy of CNIs. Both cyclosporine [47] and tacrolimus [48], given alone or in combination with corticosteroids, could obtain complete or, more frequently, partial remission of proteinuria. Relapses were frequent after withdrawal of therapy. However, many patients might be maintained in partial remission with stable renal function using low-dose CNI and regular monitoring of clinical and biological conditions. Observational reports showed that mycophenolate mofetil (MMF) often associated with prednisone could significantly reduce proteinuria. However, those studies were smallsized and had a too short follow-up to assess the possible role of mycophenolate in LMN.

In patients with severe nephrotic syndrome, we are using a combination of either cyclophosphamide or chlorambucil with glucocorticoids similar to that adopted in primary $\mathrm{MN}$ [49]. In a preliminary experience with 11 patients with LMN, 7 patients were in complete remission, and 3 patients were in partial remission after a mean follow-up of 83 months. Only one of them developed a reversible flare-up. The eleventh patient entered partial remission but developed extracapillary glomerulonephritis and eventually progressed to ESRD 24 years after the clinical onset of renal disease [50]. Rituximab has been used largely in refractory forms of proliferative lupus nephritis. In the Lupus Nephritis Assessment with Rituximab (LUNAR) study, a randomized prospective controlled trial comparing MMF to MMF plus rituximab, the addition of rituximab led to more responders and improvement in anti-dsDNA antibodies and serum complement levels. However, at 1 year, the patients getting rituximab did not experience improved clinical outcomes. The patients entered into this trial mostly had Class III or IV SLE nephritis. There were few patients with Class V LMN [51]. Chavarot et al. [52] treated 15 patients with LMN with rituximab. Complete remission was achieved in 8 patients and partial remission in 5. Two patients failed to respond. In the Rituxilup study, 50 patients $(44 \%$ of whom had pure class V lupus nephritis) were treated with 2 doses of rituximab $(1 \mathrm{~g})$ and methylprednisolone (500 $\mathrm{mg}$ ) on days 1 and 15, and maintenance treatment of MMF. By 52 weeks, complete and partial remissions had been achieved in $26(52 \%)$ and 17 patients (34\%), respectively. Twelve relapses occurred in 11 patients, and 6 patients had systemic flares. Adverse events requiring hospitalization occurred in 9 of 50 patients, in 5 because of infection [53]. A systematic analysis of the use of rituximab in refractory lupus nephritis reported that this drug achieved complete or partial response in $67 \%$ of patients with refractory LMN [54].

A few randomized, controlled trials have been performed in LMN. In a trial, 42 patients were randomized to receive prednisone alone or prednisone combined with cyclosporine for 11 months or alternate-month intravenous pulse cyclophosphamide for 6 doses. Although both cyclophosphamide and cyclosporine were more effective than prednisone in inducing remissions of proteinuria, relapse of nephrotic syndrome occurred significantly more often after completion of cyclosporine than cyclophosphamide [55]. The Aspreva Lupus Management Study (ALMS) group compared MMF versus monthly intravenous cyclophosphamide in patients with lupus nephritis. Both groups received prednisone, tapered from a maximum starting dosage of $60 \mathrm{mg} /$ day [56]. Among 370 participants, 84 patients with class $\mathrm{V}$ disease were randomized to 2 equal groups; each group had comparable entry variables, but one received MMF and the other one intravenous cyclophosphamide. Within these groups, 33 patients on MMF and 32 patients on cyclophosphamide completed 24 weeks of treatment. There were no differences between the groups in mean values for the measured end points. Similarly, no difference was found regarding the number of patients who did not complete the study or who died [57]. However, the aim of the trial was to assess the effects of 2 different treatments for induction therapy in lupus nephritis. The duration of the trial was too short to evaluate the efficacy and safety of the 2 regimens in a slowly progressive disease such as lupus MN. After the results of ALMS trial, some nephrologists prefer to use MMF for induction therapy in patients with class $\mathrm{V}$ since the drugs is safer with a lower risk of ovarian failure than cyclophosphamide. The American College of Rheumatology guidelines recommended MMF plus prednisone as the initial treatment for patients with LMN and nephrotic syndrome [58]. However, relapses are frequent after MMF withdrawal and some patients do not tolerate the drug because of gastrointestinal troubles. A randomized controlled study compared 6 months therapy of tacrolimus $(0.1 \mathrm{mg} / \mathrm{kg} /$ day $)$ versus MMF $(2 \mathrm{~g}$ per day); all patients were also given high-dose prednisolone 
$(0.6 \mathrm{mg} / \mathrm{kg} /$ day for 6 weeks tapered to $10 \mathrm{mg} /$ day for maintenance). The subgroup of 28 patients with LMN treated with tacrolimus had better improvement of proteinuria and achieved complete and partial remission in all the 16 participants in comparison with 7 out of 12 patients assigned to MMF [59]. A multi-targeted therapy, based on tacrolimus, MMF and corticosteroids, proved to be superior than intravenous cyclophosphamide for induction therapy and resulted in a low renal relapse rate and fewer adverse events in comparison with azathioprine in the maintenance therapy [60].However, only few patients with lupus class $\mathrm{V}$ were enrolled in the study.

In summary, different treatments proved to be effective in LMN, but a number of uncertainties still remain. Corticosteroids alone are poorly effective, but they may be useful in combination with other immunosuppressive drugs. In patients with severe nephrotic syndrome, reduction of proteinuria may be obtained with cyclosporine or tacrolimus, alone or combined with prednisone. However, complete remission is infrequent and many patients relapse when the drug is withdrawn. MMF or azathioprine is less effective than CNI in reducing proteinuria, but they can help maintaining remission. Cyclical therapy with corticosteroid and cyclophosphamide may obtain stable results, but the response may occur after months and their use requires a careful clinical monitoring or may be contraindicated in some patients. Rituximab is easier to manage but the optimal dosage is still to be defined; its efficacy and safety in the long-term are still poorly known. Few data are available with multitarget therapy in LMN and only in patients with Asian ethnicity. Finally, some patients who do not respond to a certain treatment may respond to a different therapy. Thus, the choice of treatment is largely individual and may be influenced by the characteristics of the patient and by the personal experience of the physician. Whatever the basic therapy, every effort should be made to early detect and treat renal flares early.

\section{Conflict of Interest Statement}

C.P. and G.M. have no conflicts of interest to declare. A.F. is an inventor on pending or issued patents (PCT/US11/56272, PCT/ US12/62594, PCT/US2019/041730, PCT/US2019/032215, PCT/ US13/36484, and PCT 62/674,897) aimed to diagnosing or treating proteinuric kidney diseases. She stands to gain royalties from her future commercialization of these patents. A.F. is the vice president of L\&F Health LLC and is a consultant for ZyVersa Therapeutics, Inc. ZyVersa Therapeutics, Inc. has licensed worldwide rights to develop and commercialize hydroxypropyl-beta-cyclodextrin from L\&F Research for the treatment of kidney disease.

\section{Funding Sources}

C.P. and G.M. did not receive any funding. A.F. is supported by the NIH grants R01DK117599, R01DK104753, R01CA227493, U54KD083912, UM1DK100846, U01DK116101, and UL1TR000460 (Miami Clinical Translational Science Institute).

\section{Author Contribution}

C.P. conceived the study. Both C.P. and G.M. equally contributed in reviewing the literature and in writing the paper. A.F. was added to improve the quality of the paper. She reviewed and corrected the manuscript and introduced new important parts.

\section{References}

1 Churg J, Bernstein J, Glassock RJ. Renal disease: classification and atlas of glomerular diseases. 2nd ed. New York, Tokyo: IgakyShoin; 1995.

2 Weening JJ, D’Agati VD, Schwartz MM, Seshan SV, Alpers CE, Appel GB, et al. The classification of glomerulonephritis in systemic lupus erythematosus revisited. J Am Soc Nephrol. 2004;15(2):241-50.

3 Kuroki A, Shibata T, Honda H, Totsuka D, Kobayashi K, SugisakiGlomerular T, et al. Glomerular and serum IgG subclasses in diffuse proliferative lupus nephritis, membranous lupus nephritis, and idiopathic membranous nephropathy. Intern Med. 2002; 41(11):936-42.
4 Beck LH Jr, Bonegio RG, Lambeau G, Beck DM, Powell DW, Cummins TD, et al. M-type phospholipase A2 receptor as target antigen in idiopathic membranous nephropathy. $\mathrm{N}$ Engl J Med. 2009;361(1):11-21.

5 Ronco P, Debiec H. Antigen identification in membranous nephropathy moves toward targeted monitoring and new therapy. J Am Soc Nephrol. 2010;21(4):564-9.

6 Garcia-Vives E, Solé C, Moliné T, AlvarezRios AM, Vidal M, Agraz I, et al. Antibodies to M-type phospholipase A2 receptor (PLA 2 $\mathrm{R})$ in membranous lupus nephritis. Lupus. 2019;28(3):396-405.

7 Tomas NM, Beck LH Jr, Meyer-Schwesinger C, Seitz-Polski B, Ma H, Zahner G, et al. Thrombospondin type-1 domain-containing 7A in idiopathic membranous nephropathy. N Engl J Med. 2014;371(24):2277-87.
8 Bayema IM, Wilhelmus S, Alpers CE, Bruijn JA, Colvin RB, Cook HT, et al. Revision of the international society of nephrology/renal pathology society classification for lupus nephritis: clarification of definitions, and modified National Institutes of Health activity and chronicity indices. Kidney Int. 2018;93(4): 789-96.

9 Petri M, Kasitanon N, Lee SS, Link K, Magder L, Bae SC, et al. Systemic lupus international collaborating clinics. Systemic lupus international collaborating clinics renal activity/response exercise: development of a renal activity score and renal response index. Arthritis Rheum. 2008;58(6):1784-8. 
10 Kwon OC, Cho YM, Oh JS, Hong S, Lee CK, Yoo B, et al. Renal flare in class $\mathrm{V}$ lupus nephritis: increased risk in patients with tubulointerstitial lesions. Rheumatol Int. 2019; 39(12):2061-7.

11 Austin HA III, Illei GG. Membranous lupus nephritis. Lupus. 2005;14(1):65-71.

12 Ponticelli C, Glassock RJ. Glomerular diseases: membranous nephropathy: a modern view. Clin J Am Soc Nephrol. 2014;9(3):60916.

13 Lech M, Anders HJ. The pathogenesis of lupus nephritis. J Am Soc Nephrol. 2013;24(9): 1357-66.

14 Bruschi M, Bonanni A, Petretto A, Vaglio A, Pratesi F, Santucci L, et al. Neutrophil extracellular traps (NETs) profiles in patients with incident SLE and lupus nephritis. J Rheumatol. 2020;47(3):377-86.

15 Arneth B. Systemic lupus erythematosus and DNA degradation and elimination defects. Front Immunol. 2019;10:1697.

16 Wang J, Dai M, Cui Y, Hou G, Deng J, Gao X, et al. Association of abnormal elevations in IFIT3 with overactive cyclic GMP-AMP synthase/stimulator of interferon genes signaling in human systemic lupus erythematosus monocytes. Arthritis Rheumatol. 2018; 70(12):2036-45.

17 Salant DJ. Unmet challenges in membranous nephropathy. Curr Opin Nephrol Hypertens. 2019;28(1):70-6.

18 Chen D, Hu W. Lupus podocytopathy: a distinct entity of lupus nephritis. J Nephrol. 2018;31(5):629-34.

19 Wang H, Xu J, Zhang X, Ren YL, Cheng M, Guo ZL, et al. Tubular basement membrane immune complex deposition is associated with activity and progression of lupus nephritis: a large multicenter Chinese study. Lupus. 2018;27(4):545-55.

20 Kalaaji M, Fenton KA, Mortensen ES, Olsen R, Sturfelt G, Alm P, et al. Glomerular apoptotic nucleosomes are central target structures for nephritogenic antibodies in human SLE nephritis. Kidney Int. 2007;71(7):664-72.

$21 \mathrm{MaH}$, Sandor DG, Beck LH. The role of complement in membranous nephropathy. Semin Nephrol. 2013;33(6):531-42.

22 Schmiedeke TM, Stöckl FW, Weber R, Sugisaki Y, Batsford SR, Vogt A. Histones have high affinity for the glomerular basement membrane. Relevance for immune complex formation in lupus nephritis. J Exp Med. 1989;169(6):1879-94.

23 Song D, Guo WY, Wang FM, Li YZ, Song Y, Yu F, et al. Complement alternative pathway's activation in patients with lupus nephritis. Am J Med Sci. 2017;353(3):247-57.

24 Cybulsky AV, Takano T, Papillon J, Khadir A, Liu J, Peng H. Complement C5b-9 membrane attack complex increases expression of endoplasmic reticulum stress proteins in glomerular epithelial cells. J Biol Chem. 2002;277(44): 41342-51.
25 Nangaku M, Couser WG. Mechanisms of immune-deposit formation and the mediation of immune renal injury. Clin Exp Nephrol. 2005;9(3):183-91.

26 Shimizu S, Sugiyama N, Masutani K, Sadanaga A, Miyazaki Y, Inoue Y, et al. Membranous glomerulonephritis development with Th2type immune deviations in MRL/lpr mice deficient for IL-27 receptor (WSX-1). J Immunol. 2005;175(11):7185-92.

27 Mok CC. Membranous nephropathy in systemic lupus erythematosus: a therapeutic enigm. Nat Rev Nephrol. 2009;5:212-20.

28 Moroni G, Depetri F, Ponticelli C. Lupus nephritis: when and how often to biopsy and what does it mean? J Autoimmun. 2016;74: 27-40.

29 Ayoub I, Cassol C, Almaani S, Rovin B, Parikh SV. The kidney biopsy in systemic lupus erythematosus: a view of the past and a vision of the future. Adv Chronic Kidney Dis. 2019; 26(5):360-8.

30 Sloan RP, Schwartz MM, Korbet SM, Borok RZ. Long-term outcome in systemic lupus erythematosus membranous glomerulonephritis. Lupus nephritis collaborative study group. J Am Soc Nephrol. 1996;7(2):299-305.

31 Mok CC, Ying KY, Lau CS, Yim CW, Ng WL, Wong WS, et al. Treatment of pure membranous lupus nephropathy with prednisone and azathioprine: an open-label trial. Am J Kidney Dis. 2004:43:269-76.

32 Mercadal L, Montcel ST, Nochy D. The Groupe D'Etudes Nephrologiques en Ile de France. Factors affecting outcome and prognosis in membranous lupus nephropathy. Nephrol Dial Transplant. 2002;17:1771-8.

33 Moroni G, Quaglini S, Gravellone L, Gallelli B, Leoni A, Messa P, et al. Membranous nephropathy in systemic lupus erythematosus: long-term outcome and prognostic factors of 103 patients. Semin Arthritis Rheum. 2012; 41(5):642-51.

34 Moroni G, Quaglini S, Maccario M, Banfi G, Ponticelli C. "Nephritic flares" are predictors of bad long-term renal outcome in lupus nephritis. Kidney Int. 1996;50(6):2047-53.

35 Parikh SV, Nagaraja HN, Hebert L, Rovin BH. Renal flare as a predictor of incident and progressive CKD in patients with lupus nephritis. Clin J Am Soc Nephrol. 2014;9(2):279-84.

36 Narváez J, Ricse M, Gomà M, Mitjavila F, Fulladosa X, Capdevila O, et al. The value of repeat biopsy in lupus nephritis flares. Medicine. 2017;96(24):e7099.

37 Glassock RJ. Prophylactic anticoagulation in nephrotic syndrome: a clinical conundrum. J Am Soc Nephrol. 2007;18(8):2221-5.

38 Zelniker TA, Wiviott SD, Raz I, Im K, Goodrich EL, Bonaca MP, et al. SGLT2 inhibitors for primary and secondary prevention of cardiovascular and renal outcomes in type 2 diabetes: a systematic review and meta-analysis of cardiovascular outcome trials. Lancet. 2019;393(10166):31-9.
39 Mok CC, Birmingham DJ, Ho LY, Hebert LA, Song $\mathrm{H}$, Rovin BH. Vitamin D deficiency as marker for disease activity and damage in systemic lupus erythematosus: a comparison with anti-dsDNA and anti-C1q. Lupus. 2012; 21(1):36-42.

40 Vieth R. Vitamin D supplementation, 25-hydroxyvitamin D concentrations, and safety. Am J Clin Nutr. 1999;69(5):842-56.

41 Sarasin FP, Schifferli JA. Prophylactic oral anticoagulation in nephrotic patients with idiopathic membranous nephropathy. Kidney International. 1994;45(2):578-85.

42 Derebail VK, Rheault MN, Kerlin BA. Role of direct oral anticoagulants in patients with kidney disease. Kidney Int. 2020;97(4):664-75.

43 KDIGO clinical practice guidelines for glomerulonephritis. Kidney Int 2012; 2. 156-62

44 Hofstra JM, Wetzels JFM. Should aspirin be used for primary prevention of thrombotic events in patients with membranous nephropathy? Kidney Int. 2016;89(5):981-3.

45 Fanouriakis A, Kostopoulou M, Cheema K, Anders HJ, Aringer M, Bajema I, et al. 2019 update of the Joint European League Against Rheumatism and European Renal Association-European Dialysis and Transplant Association (EULAR/ERA-EDTA) recommendations for the management of lupus nephritis. Ann Rheum Dis. 2020;79(6):713-23.

46 Ponticelli C, Moroni G. Hydroxychloroquine in systemic lupus erythematosus (SLE). Expert Opin Drug Saf. 2017;16(3):411-9.

47 Hallegua D, Wallace DJ, Metzger AL, Rinaldi RZ, Klinenberg JR. Cyclosporine for lupus membranous nephritis: experience with ten patients and review of the literature. Lupus. 2000;9(4):241-51.

48 Szeto CC, Kwan BC, Lai FM, Tam LS, Li EK Chow KM, et al. Tacrolimus for the treatment of systemic lupus erythematosus with pure class V nephritis. Rheumatology. 2008;47(11): 1678-81.

49 Ponticelli C, Altieri P, Scolari F, Passerini P, Roccatello D, Cesana B, et al. A randomized study comparing methylprednisolone plus chlorambucil versus methylprednisolone plus cyclophosphamide in idiopathic membranous nephropathy. J Am Soc Nephrol. 1998;9(3):444-50.

50 Moroni G, Maccario M, Banfi G, Quaglini S, Ponticelli C. Treatment of membranous lupus nephritis. Am J Kidney Dis. 1998;31(4): 681-6.

51 Rovin BH, Furie R, Latinis K, Looney RJ, Fervenza FC, Sanchez-Guerrero J, et al. Efficacy and safety of rituximab in patients with active proliferative lupus nephritis: the Lupus $\mathrm{Ne}$ phritis Assessment with Rituximab study. Arthritis Rheum. 2012;64(4):1215-26.

52 Chavarot N, Verhelst D, Pardon A, Caudwell V, Mercadal L, Sacchi A, et al. Groupe Coopératif sur le Lupus Rénal. Rituximab alone as induction therapy for membranous lupus nephritis: a multicenter retrospective study. Medicine. 2017;96(27):e7429. 
53 Condon MB, Ashby D, Pepper RJ, Cook HT, Levy JB, Griffith M, et al. Prospective observational single-centre cohort study to evaluate the effectiveness of treating lupus nephritis with rituximab and mycophenolate mofetil but no oral steroids. Ann Rheum Dis. 2013; 72(8):1280-6.

54 Weidenbusch M, Römmele C, Schröttle A, Anders HJ. Beyond the LUNAR trial. Efficacy of rituximab in refractory lupus nephritis. Nephrol Dial Transplant. 2013;28(1):106-11.

55 Austin HA III, Gabor G, Illei GG, Braun MJ, Balow JE. Randomized, controlled trial of prednisone, cyclophosphamide, and cyclosporine in lupus membranous nephropathy. J Am Soc Nephrol. 2009;20(4):901-11.
56 Appel GB, Contreras G, Dooley A, Ginzler EM, Isenberg D, Jayne D, et al. Aspreva lupus management study group. Mycophenolate mofetil cyclophosphamide for induction treatment of lupus nephritis. J Am Soc Nephrol. 2009;20(5):1103-12.

57 Radhakrishnan J, Moutzouris DA, Ginzler EM, Solomons N, Siempos II, Appel GB. Mycophenolate mofetil and Intravenous cyclophosphamide are similar as induction therapy for class V lupus nephritis. Kidney Int. 2010; 77(2):152-60.
58 Hahn BH, McMahon MA, Wilkinson A, Wallace WD, Daikh DI, Fitzgerald JD, et al. American College of Rheumatology guidelines for screening, treatment and management of lupus nephritis. Arthritis Care Res. 2012;64:797-808.

59 Mok CC, Ying KY, Yim CW, Siu YP, Tong $\mathrm{KH}$, To $\mathrm{CH}$, et al. Tacrolimus versus mycophenolate mofetil for induction therapy of lupus nephritis: a randomised controlled trial and long-term follow-up. Ann Rheum Dis. 2016;75(1):30-6.

60 Zhang H, Liu Z, Zhou M, Liu Z, Chen J, Xing $\mathrm{C}$, et al. Multitarget therapy for maintenance treatment of lupus nephritis. J Am Soc Nephrol. 2017;28(12):3671-8. 